\title{
Importância do tempo de espera pós-tratamento clareador no selamento marginal de restaurações classe V em resina composta
}

\begin{abstract}
- Beatriz Togoro Ferreira da Silva Department of Restorative Dentistry, School of Dentistry, University of São Paulo, São Paulo, SP, Brazil - Alessandra Pereira de Andrade Department of Restorative Dentistry, School of Dentistry, University of São Paulo, São Paulo, SP, Brazil • Cynthia Soares de Azevedo Department of Restorative Dentistry, School of Dentistry, University of São Paulo, São Paulo, SP, Brazil • Rubens Côrte Real de Carvalho Department of Restorative Dentistry, School of Dentistry, University of São Paulo, São Paulo, SP, Brazil • Adriana Bona Matos Department of Restorative Dentistry, School of Dentistry, University of São Paulo, São Paulo, SP, Brazil
\end{abstract}

RESUMO | O objetivo deste estudo foi avaliar o conteúdo mineral do esmalte submetido ao clareamento dental e a influência do tempo de espera após o tratamento clareador no selamento marginal de restaurações classe $\mathrm{V}$ em resina composta. Neste estudo, a variável de resposta selamento marginal foi avaliada por metodologia de microinfiltração, segundo o fator de variação tempo de espera para o procedimento restaurador, imediatamente após, e 7 e 14 dias após o tratamento clareador. O conteúdo mineral foi avaliado pelo método QLF (quantitative light-induced fluorescence). As unidades experimentais foram compostas por 40 coroas de incisivos bovinos que foram distribuídas entre os 4 grupos experimentais $(\mathrm{n}=10)$ : G1, dentes bovinos não clareados (controle); G2, dentes clareados e imediatamente restaurados; G3, dentes clareados e restaurados após 7 dias; G4, dentes clareados e restaurados após 14 dias. Os escores de infiltração foram analisados por 3 examinadores previamente calibrados. Não foi observada diferença estatística entre os grupos com relação à alteração mineral do substrato clareado (ANOVA para mensurações repetidas, $p=0,2130$ ) e ao grau de microinfiltração marginal (Friedman, $p=0,2551$ ). Pôde-se concluir que o protocolo clareador utilizado é seguro por não acarretar alterações minerais do esmalte clareado, e que o tempo de espera para a realização de procedimentos adesivos não interferiu no selamento marginal de restaurações em resina composta.

DESCRITORES | Clareamento Dental; Resinas Compostas; Infiltração Dentária.

ABSTRACT | Importance of post-bleaching time interval on the marginal seal of class $\mathbf{V}$ composite resin restorations • The aim of this study was to evaluate changes in mineral content of bleached enamel and the influence of post-bleaching time interval on the marginal seal of class V composite restorations. Forty bovine incisors were restored using Magic Bond adhesive and Fill NT Premium composite according to the manufacturer's instructions. The teeth were randomly divided into 4 experimental groups $(\mathrm{n}=10)$ : G1, not bleached (control); G2, bleached and immediately restored; G3, bleached and restored after 7 days; G4, bleached and restored after 14 days. The mineral content was evaluated by QLF (quantitative light-induced fluorescence). The microleakeage scores were analyzed by 3 calibrated examiners. There was no statistical difference between the groups regarding change of the bleached mineral substrate (repeated measures ANOVA, $p=0.2130$ ) and the microleakage scores (Friedman, $\mathrm{p}=0.2551$ ). It was concluded that the bleaching protocol used is safe because it does not cause mineral changes in the bleached enamel, and that the post-bleaching time interval observed before performing adhesive procedures did not affect the marginal seal of composite resin restorations.

DESCRIPTORS || Tooth Bleaching; Composite Resins; Dental Leakeage.

CORRESPONDING AUTHOR | Adriana Bona Matos Departament of Restorative Dentistry, School of Dentistry, University of São Paulo • Av. Professor Lineu Prestes, 2227 São Paulo, SP, Brazi • 05508-000 E-mail: bona@usp.br

- Received Jul 15, 2013 • Accepted Sep 11, 2013

- Dol http://dx.doi.org/10.11606/issn.2357-8041.v20i2p74-81 


\section{INTRODUÇÃO}

A demanda estética dos pacientes impulsiona o interesse em realizar estudos sobre o clareamento dental, seus efeitos sobre o substrato dental e sobre a qualidade de restaurações estéticas realizadas com resinas compostas. ${ }^{22}$

O clareamento de dentes vitalizados ocorre graças à interação físico-química entre os tecidos dentais mineralizados e o agente clareador. ${ }^{8} \mathrm{O}$ peróxido de hidrogênio, ingrediente ativo dos géis clareadores, é um forte oxidante que possui a capacidade de se difundir livremente através do esmalte e da dentina em função da permeabilidade desses substratos e devido ao baixo peso molecular dessas substâncias. Assim o clareamento dental ocorre graças à permeabilidade do esmalte e da dentina $\mathrm{e}$ à difusibilidade dos agentes clareadores.,15

O clareamento dental consiste na degradação de moléculas de alto peso molecular, situadas no interior dos tecidos dentais mineralizados, que refletem determinado comprimento de onda de luz que incidem sobre o dente, e que fazem com que o dente pareça escurecido. A formação de cadeias moleculares longas e complexas no interior da estrutura dental é responsável pelo aumento do índice de absorção de luz pelo elemento dental. Assim moléculas com dimensões reduzidas e peso molecular menor originadas pelo clareamento dental permitem o restabelecimento das propriedades ópticas do elemento dental. 7,15

Os resultados obtidos por estudos realizados por diferentes metodologias comprovaram a ação desmineralizadora dos agentes clareadores sobre o esmalte dental humano., ${ }^{1,8,29,30,34} \mathrm{O}$ grau de severidade dessa ação desmineralizadora pode estar relacionada ao tempo de contato do agente clareador com a superfície dental, posto que o processo de clareamento dental ocorre quando do íntimo contato entre clareador e estrutura dental.

Outros estudos ${ }^{3-6}$ avaliaram os efeitos do tratamento clareador realizado previamente à execu- ção de restaurações adesivas e demonstraram uma diminuição da resistência de união entre material restaurador e substrato dental clareado.

Outro fator que apresenta controvérsia na literatura é com relação ao tempo de espera após o término do tratamento clareador e o momento de realização de restaurações adesivas. Alguns estudos mostraram um aumento no índice de microinfiltração na dentina após o clareamento, e atribuem esse efeito à presença de oxigênio residual, proveniente do agente clareador, e a mudanças estruturais na composição do esmalte e da dentina, afetando o vedamento da interface resina/dente..$^{10}$ Entretanto, outros estudos não encontraram influência da utilização prévia de gel clareador em restaurações em resina composta. ${ }^{18,32}$

Dessa forma, considera-se importante estudar os efeitos do tratamento clareador assim como o tempo de espera para a execução do procedimento restaurador em dentes clareados, a fim de se otimizar o vedamento marginal e consequentemente reduzir o risco de microinfiltração.

Assim o objetivo deste estudo foi avaliar o conteúdo mineral do esmalte submetido ao clareamento dental e a influência do tempo de espera após o tratamento clareador no selamento marginal de restaurações classe $\mathrm{V}$ em resina composta.

\section{MATERIAL E MÉTODOS Delineamento experimental}

Neste estudo, a variável resposta selamento marginal foi avaliada por metodologia de microinfiltração, segundo o fator de variação tempo de espera para o procedimento restaurador, imediatamente após, e 7 e 14 dias após o tratamento clareador. As unidades experimentais foram compostas por 40 coroas de incisivos bovinos distribuídas entre 4 grupos experimentais $(n=10)$ :

- G1, dentes bovinos não clareados (controle);

- G2, dentes clareados e imediatamente restaurados; 
- G3, dentes clareados e restaurados após 7 dias;

- G4, dentes clareados e restaurados após 14 dias.

\section{Seleção da amostra}

Os dentes receberam uma profilaxia e foram observados com o auxílio de uma lupa estereoscópica com uma lente de aumento de 40 vezes com o objetivo de verificar a ausência de defeitos, trincas e/ou imperfeições no esmalte (Figura 1A). Todos os elementos dentais foram analisados pelo método de fluorescência do substrato dental $\left(\mathrm{QLF}^{\mathrm{m}}\right.$, Quantitative Light-induced Fluorescence System, Inspektor Reserch Systems BV, Amsterdam, The Netherlands). Essa análise teve como objetivo verificar se algum dos 40 elementos dentais apresentava alguma área de desmineralização em suas faces vestibulares que pudessem causar alguma interferência nos resultados deste estudo (Figura 1A). O método de quantificação mineral por fluorescência do tecido dental baseia-se em diferenças ópticas entre esmalte sadio e esmalte desmineralizado e na propriedade intrínseca da estrutura dental de apresentar fluorescência quando iluminada por fonte de luz ultravioleta e visível na região do azul-verde e vermelho do espectro eletromagnético. É possível diferenciar a estrutura sadia da estrutura desmineralizada com os comprimentos de onda na região do azul-violeta, pois a fluorescência é menor nas áreas em que há perda mineral, sendo observadas como manchas escuras na imagem. . $^{9,19,33}$

Para a obtenção das imagens, os espécimes foram secos com jato de ar comprimido por 30 segundos para padronização do grau de desidratação do esmalte desmineralizado. O dispositivo intraoral e a ponta da peça de mão do equipamento foram posicionados no suporte para estudos. Esse suporte possibilita que o espécime analisado e a peça de mão permaneçam dispostos paralelos entre si, permitindo que a distância entre eles possa ser ajustada a fim de propiciar uma condição de foco ideal, e para que a distância entre o espécime analisado e a peça de mão seja a mesma em todas as mensurações. As imagens foram capturadas em câmara escura, armazenadas no disco rígido da unidade principal do equipamento $\mathrm{QLF}^{\mathrm{TM}}$. As imagens obtidas foram analisadas, por meio do aplicativo do equipamento (Inspektor ${ }^{\text {TM }}$ PRO, Inspektor Dental Care, Amsterdam, The Netherlands), quanto à área da lesão $\left(\mathrm{mm}^{2}\right)$ e à profundidade da lesão, expressa em percentual de perda de fluorescência do tecido $(\Delta \mathrm{F}$ em $\%)$. O volume da lesão ( $\Delta \mathrm{Q}$ em $\mathrm{mm}^{2} \%$ ) é o índice final relativo ao valor de perda mineral do substrato analisado. Foram selecionados apenas os elementos que apresentaram a face vestibular sem áreas de desmineralização $(\Delta \mathrm{Q}=0)$.

\section{Preparo dos espécimes e tratamento clareador}

As coroas dentais foram recobertas por uma fita adesiva de dimensões de $5 \times 5 \mathrm{~mm}$ em sua porção cervical e por uma camada de verniz ácidoresistente (esmalte cosmético incolor, Maybelline Colorama, São Paulo, SP, Brasil; Figura 1B). Após a secagem do verniz por 24 horas, a fita adesiva foi removida dos fragmentos, delimitando uma área de $25 \mathrm{~mm}^{2}$ onde o tratamento clareador com peróxido de carbamida a 10\% (Opalescence 10\% regular, Ultradent Product Inc., South Jordan, UT, EUA) foi executado durante 21 dias consecutivos (Figura 1C). Os espécimes foram armazenados em saliva artificial (Saliform, Fórmula e Ação, São Paulo, Brasil) durante o período de clareamento. A saliva foi substituída diariamente. Essa delimitação permite a obtenção de uma área não tratada que desempenha a função de controle negativo do estudo. Uma nova avaliação do conteúdo mineral por meio do método $\mathrm{QLF}^{\mathrm{TM}}$ foi realizada na mesma área em que a leitura foi realizada previamente ao clareamento (Figura 1D), e os dados de $\Delta \mathrm{Q}$ foram posteriormente submetidos a análise estatística pelo teste de ANOVA para mensurações repetidas $(\alpha=5 \%)$. 
Figura 1 | Metodologia laboratorial utilizada.

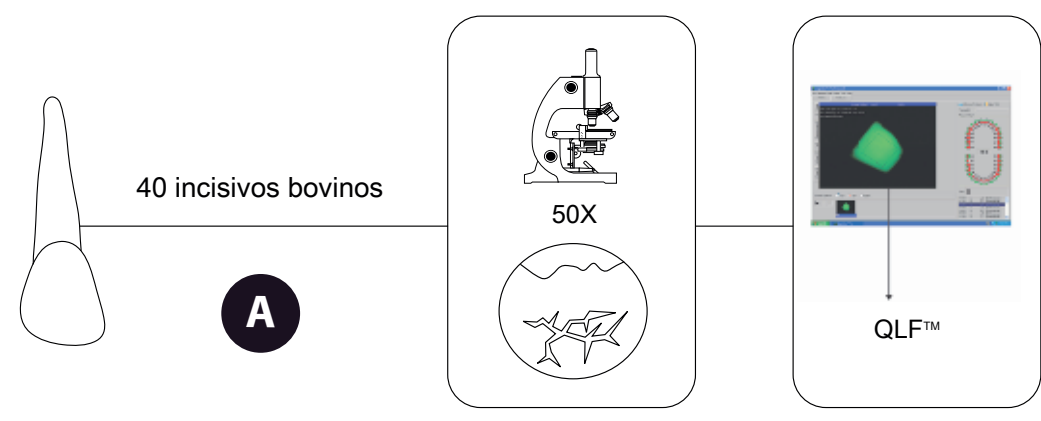

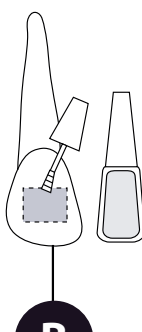

B

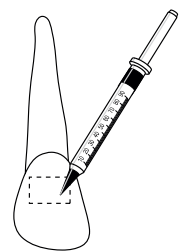

C
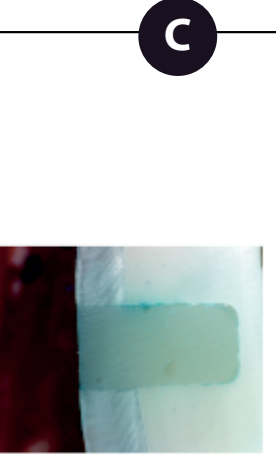

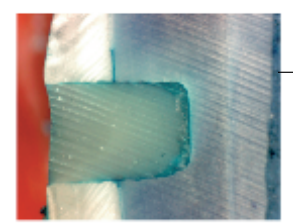

Análise dos escores de microinfiltração
Após o término do período de clareamento dental, os espécimes receberam restaurações adesivas conforme os diferentes tempos de espera:

- imediatamente após o tratamento clareador (G2),

- 7 dias após o término do tratamento clareador (G3) e

- 14 dias após o tratamento clareador (G4).

Os espécimes permaneceram armazenados em saliva artificial durante os tempos de espera e todas as etapas do experimento.

\section{Procedimento restaurador e ensaio de microinfiltração}

Foram realizados preparos cavitários classe V na superfície vestibular de cada coroa dental com dimensões de $2 \mathrm{~mm}$ de altura, $4 \mathrm{~mm}$ de largura e $2 \mathrm{~mm}$ de profundidade. Em seguida foi realizado o procedimento adesivo com um sistema do tipo condicione-e-lave (Magic Bond, Vigodent, Rio de Janeiro, Brasil) e a inserção em incrementos da resina composta (Fill magic NT Premium, Vigodent, Rio de Janeiro, Brasil) conforme as instruções do fabricante (Figura 1E). Os espécimes restaurados perma- 
Tabela 1 | Valores médios ( \pm desvio-padrão) obtidos para a análise do conteúdo mineral dos grupos experimentais $(\alpha=5 \%)$

\begin{tabular}{c|c|c|c|c|c|c|c}
\multicolumn{9}{c|}{ Valores de $\Delta$ Q em mm² } \\
\hline T0 & T1 & T0 & T1 & T0 & T1 & T0 & T1 \\
\hline $0,0^{\mathrm{a}}$ & $0,0^{\mathrm{a}}$ & $0,0^{\mathrm{a}}$ & $0,001^{\mathrm{a}}$ & $0,0^{\mathrm{a}}$ & $0,001^{\mathrm{a}}$ & $0,0^{\mathrm{a}}$ & $0,001^{\mathrm{a}}$ \\
\hline$(0,0)$ & $(0,0)$ & $(0,0)$ & $(0,0001)$ & $(0,0)$ & $(0,0001)$ & $(0,0)$ & $(0,0001)$ \\
\hline
\end{tabular}

TO = pré-clareamento dental; T1 = pós-clareamento dental. neceram armazenados em um umidificador a $37^{\circ} \mathrm{C}$ durante 24 horas para posteriormente receberem polimento e acabamento. Após serem submetidos a 500 ciclos térmicos de $5^{\circ} \mathrm{C}$ e $55^{\circ} \mathrm{C}^{2}$ os espécimes foram recobertos com verniz ácido-resistente (esmalte cosmético vermelho, Maybelline Colorama, São Paulo, SP, Brasil) de modo que permanecesse uma janela de dimensões $3 \mathrm{~mm}$ de altura e $5 \mathrm{~mm}$ de largura, com a exposição de toda a margem da restauração realizada, para posteriormente serem imersos em solução de azul de metileno a $2 \%$ por 24 horas a $37^{\circ} \mathrm{C}^{12}$ e lavados em água corrente por $15 \mathrm{minu}$ tos (Figura $1 \mathrm{~F}$ ). Os espécimes foram seccionados no sentido vestíbulo-lingual na porção central da restauração utilizando disco diamantado. As imagens dos espécimes seccionados foram registradas com auxílio de um microscópio digital em aumento de 50 vezes (MiView USB Digital Microscope, Chinavasion Wholesale, Guangdong, China) para a análise de 3 examinadores (A, B e C) previamente calibrados.

Foram atribuídos os seguintes escores para penetração do corante:

- $\mathrm{o}=$ sem presença de corante na interface dente/ restauração;

- 1 = presença do corante atingindo a parede lateral dos preparos cavitários;

- 2 = presença do corante atingindo a parede axial dos preparos cavitários.

Os escores de infiltração foram analisados estatisticamente pelo teste de Kappa (5\%) para análise da concordância interexaminador e teste de Friedman $(5 \%)$ para verificação de diferenças estatísti-
Tabela 2 | Coeficiente Kappa de concordância para avaliação do teste interexaminador.

\begin{tabular}{c|c|c} 
Concordância & Coeficiente Kappa & $\begin{array}{c}\text { Conclusão de } \\
\text { replicabilidade }\end{array}$ \\
\hline$A \times B$ & $0.7562(p<0,0001)$ & excelente \\
\hline$A \times C$ & $0.9180(p<0,0001)$ & excelente \\
\hline$B \times C$ & $0.9083(p<0,0001)$ & excelente \\
\hline
\end{tabular}

cas entre os grupos experimentais em relação ao grau de microinfiltração.

\section{RESULTADOS}

Para a avaliação de conteúdo mineral pelo método QLF $^{\mathrm{TM}}$ por meio do teste ANOVA para mensurações repetidas, observou-se que não houve diferença estatística entre o conteúdo mineral antes e após o clareamento dental $(p=0,2130)$, notando-se que o tratamento clareador realizado não acarretou perdas minerais do esmalte clareado. As médias e os desvios-padrão podem ser observados na Tabela 1.

Para o teste de concordância interexaminador, os resultados obtidos podem ser observados na Tabela 2 .

O teste de Friedman revelou não haver diferença estatística entre os quatro grupos analisados em relação ao grau de microinfiltração ( $p=0,2551$; Figura 2).

\section{DISCUSSÃO}

Qualidade e longevidade são as características almejadas na união entre material restaurador e substrato dental. São esses elementos que indicam o sucesso das restaurações estéticas adesivas. ${ }^{26} \mathrm{~A}$ 


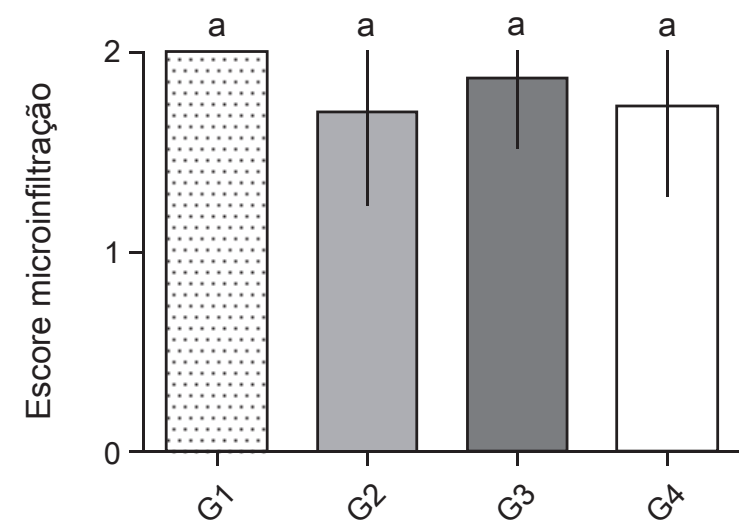

G1 - controle

$\mathrm{G} 2$ - clareados e imediatamente restaurados

G3 - clareados e restaurados após 7 dias

G4 - clareados e restaurados após 14 dias

0 - sem presença de corante na interface dente/restauração

1 - presença do corante atingindo a parede lateral dos preparos cavitários

2 - presença do corante atingindo a parede axial dos preparos cavitários

Figura 2 | Diferenças estatísticas da microinfiltração entre os grupos experimentais (ANOVA 5\%).

durabilidade das restaurações diretas está intimamente relacionada ao vedamento do ângulo cavosuperficial de preparos cavitários. ${ }^{25}$

O mecanismo de adesão ao esmalte e à dentina consiste basicamente em um processo de substituição dos minerais removidos dos tecidos dentais duros pelos monômeros resinosos, obtendo-se uma retenção micromecânica pela penetração desses monômeros nas microrretenções criadas com a remoção desses minerais.

Essa zona de interdifusão entre a camada de adesivo e a dentina desmineralizada subjacente foi chamada de camada híbrida. A aplicação do ácido fosfórico, dos sistemas adesivos tipo condicionee-lave, à dentina promove uma desmineralização superficial, expondo uma trama de fibras colágenas que posteriormente é infiltrada pelo adesivo. ${ }^{23}$

A adesão ao esmalte está diretamente relacionada ao selamento marginal das restaurações adesivas. O ácido fosfórico altera a topografia do esmalte criando microporosidades e aumentando a energia de superfície que permite a penetração do compo- nente resinoso nas porosidades, formando os prolongamentos resinosos que conferem adesão entre esmalte e resina. ${ }^{11}$

Estudos que observaram menores valores de resistência adesiva quando da realização de restaurações imediatamente após o término do tratamento clareador justificam esse achado com base na ocorrência de mudanças na composição química e/ou estrutural da superfície de esmalte. ${ }^{11,16,20}$ Contudo, neste estudo não foram verificadas alterações da composição química do esmalte clareado; assim, as restaurações em resina composta realizadas imediatamente após o tratamento clareador apresentaram o mesmo grau de selamento marginal que o apresentado pelas restaurações realizadas 7 e 14 dias após o término do clareamento dental.

A maioria das pesquisas ${ }^{6,21}$ que investigam a correlação entre materiais resinosos e os tecidos dentais expostos aos agentes clareadores são estudos de resistência adesiva. Porém, a correlação inversamente direta entre o teste de resistência de união e microinfiltação marginal não pode ser estabelecida; ${ }^{14}$ assim, estudos utilizando metodologias que avaliem o selamento marginal são necessários.

Porém, este selamento marginal das restaurações em resina composta difere de acordo com o sistema adesivo utilizado. ${ }^{35}$ Neste estudo foi observada uma taxa de microinfiltração elevada para todos os grupos experimentais, incluindo o grupo controle, sendo que mais de $80 \%$ das restaurações apresentaram infiltração marginal em dentina.

Um estudo avaliando várias marcas comerciais de sistemas adesivos demonstrou que o sistema adesivo utilizado neste estudo apresentou um dos maiores índices de infiltração marginal em restaurações classe V. ${ }^{27}$ Esses resultados concordam com os do presente estudo, em que mesmo o grupo controle (sem tratamento restaurador) apresentou altos escores de infiltração marginal.

Os resultados deste estudo estão concordes com outros estudos ${ }^{17,18}$ que avaliaram o vedamento mar- 
ginal de cavidades classe $\mathrm{V}$ restaurados com sistemas adesivos e resinas compostas em elementos dentais previamente submetidos ao tratamento clareador.

Alterações microestruturais no esmalte e na dentina decorrentes do tratamento clareador, subprodutos residuais do agente clareador, tempo de aplicação e concentração e tempo podem comprometer a eficiência do vedamento marginal, acarretando infiltração e o insucesso do procedimento restaurador. ${ }^{10}$

A avaliação do conteúdo mineral pelo método de $\mathrm{QLF}^{\mathrm{TM}}$ realizada neste estudo revelou não ter havido perda mineral do esmalte dental decorrente do procedimento clareador. Outros estudos ${ }^{13,24,30,31}$ conduzidos por outras metodologias tiveram achados semelhantes quando da utilização de agentes clareadores com peróxido de carbamida a $10 \%$, a mesma concentração e o mesmo princípio ativo do agente utilizado no presente estudo.

A evidência de não ter ocorrido desmineralização do esmalte dental e, consequentemente, a ausência de alteração da morfologia do esmalte clareado também pode ter colaborado para os grupos submetidos ao clareamento dental terem se com-

\section{REFERÊNCIAS}

1. Andrade AP, Shimaoka AM, Carvalho RCR. Estudo bioquímico do esmalte dental humano tratado com agentes clareadores com diferentes concentrações de peróxido e exposto a repetidas aplicações. RPG Rev Pós-Grad. 2009 jan.-mar.;16(1):7-12.

2. Attar N, Korkmaz Y, Ozel E, Bicer CO, Firatli E. Microleakage of class $\mathrm{V}$ cavities with different adhesive systems prepared by a diamond instrument and different parameters of Er:YAG laser irradiation. Photomed Laser Surg. 2008 Dec;26(6):58591.

3. Barcellos DC, Benetti P, Fernandes W Jr, Valera MC. Effect of carbamide peroxide bleaching gel concentration on the bond strength of dental substrates and resin composite. Oper Dent. 2010 Jul-Aug;35(4):463-9.

4. Braz R, Cordeiro-Loretto S, de Castro-Lyra AM, Dantas DC, Ribeiro AI, Guênes GM, et al. Effect of bleaching on shear bond portado semelhantemente ao grupo controle.

É de fundamental importância a realização de outros estudos para a avaliação de outras técnicas e agentes de clareamento dental e o emprego de diferentes materiais restauradores para avaliação da interação entre esses dois procedimentos estéticos.

\section{CONCLUSÃO}

Dentro das limitações da metodologia utilizada neste estudo e frente aos resultados obtidos, pôdese concluir que o protocolo clareador utilizado é seguro por não acarretar alterações minerais do esmalte clareado e que o tempo de espera para realização de procedimentos adesivos não interferiu no selamento marginal de restaurações em resina composta.

\section{AGRADECIMENTOS}

Agradecemos à FAPESP (Fundação de Amparo à Pesquisa do Estado de São Paulo) pelo fomento na modalidade Auxílio Pesquisa Regular FAPESP, processo 2006/o1177-8) para a aquisição do equipamento QLF ${ }^{\mathrm{TM}}$.

strength to dentin of etch-and-rinse and self-etching primer adhesives. Acta Odontol Latinoam. 2012;25(1):20-6.

5. Can-Karabulut DC, Karabulut B. Influence of activated bleaching on various adhesive restorative systems. J Esthet Restor Dent. 2011 Dec;23(6):399-408.

6. Cavalli V, Reis AF, Giannini M, Ambrosano GM. The effect of elapsed time following bleaching on enamel bond strength of resin composite. Oper Dent. 2001 Nov-Dec;26(6):597-602.

7. Dahl JE, Pallesen U. Tooth bleaching--a critical review of the biological aspects. Crit Rev Oral Biol Med. 2003;14(4):292304.

8. Efeoglu N, Wood D, Efeoglu C. Microcomputerised tomography evaluation of $10 \%$ carbamide peroxide applied to enamel. J Dent. 2005 Aug;33(7):561-7.

9. Elton V, Cooper L, Higham SM, Pender N. Validation of enamel erosion in vitro. J Dent. 2009 May;37(5):336-41. 
10. Fayad MVL, Anbinder AL, Marques AP, Amore R, Valera MC, Araújo MAM. Avaliação da infiltração marginal após clareamento dental e restauração com resina composta, variando o sistema adesivo. PGR: Pós-Grad Rev Fac Odontol São José dos Campos 2002 jan.-abr.;5(1):43-9.

11. Garcia MG, Bonifácio CC, Carvalho RCR. Avaliação da resistência de união de resina composta ao esmalte bovino clareado com peróxido de carbamida. RPG Rev Pós-Grad. 2006 jan.-mar.;13(1):56-62.

12. Giachetti L, Scaminaci Russo D, Bambi C, Nieri M, Bertini F. Influence of operator skill on microleakege of total-etch and self-etch bonding systems. J Dent. 2008 Jan;36(1):49-53.

13. Haywood VB, Leech T, Heymann HO, Crumpler D, Bruggers K. Nightguard vital bleaching: effects on enamel surface texture and diffusion. Quintessence Int. 1990 Oct;21(10):801-4.

14. Hilton TJ. Can modern restorative procedures and materials reliably seal cavities? In vitro investigations. Part 2. Am J Dent. 2002 Aug;15(4):279-89.

15. Joiner A. Review of the effects of peroxide on enamel and dentine properties. J Dent. 2007 Dec;35(12):889-96.

16. Josey AL, Meyers IA, Romaniuk K, Symons AL. The effect of a vital bleaching technique on enamel surface morphology and the bonding of composite resin to enamel. J Oral Rehabil. 1996 Apr;23(4):244-50.

17. Khoroushi M, Fardashtaki SR. Effect of light-activated bleaching on the microleakage of Class $\mathrm{V}$ tooth-colored restorations. Oper Dent. 2009 Sep-Oct;34(5):565-70.

18. Klukowska MA, White DJ, Gibb RD, Garcia-Godoy F, GarciaGodoy C, Duschner H. The effects of high concentration tooth whitening bleaches on microleakage of Class $\mathrm{V}$ composite restorations. J Clin Dent. 2008;19(1):14-7.

19. Kühnisch J, Heinrich-Weltzien R. Quantitative light-induced fluorescence (QLF) -- a literature review. Int J Comput Dent. 2004 Oct;7(4):325-38.

20. Lai SC, Tay FR, Cheung GS, Mak YF, Carvalho RM, Wei SH, et al. Reversal of compromised bonding in bleached enamel. J Dent Res. 2002 Jul;81(7):477-81.

21. Lima AF, Sasaki RT, Araújo LS, Gaglianone LA, Freitas MS, Aguiar FH, et al. Effect of tooth bleaching on bond strength of enamel-dentin cavities restored with silorane- and dimethacrylate-based materials. Oper Dent. 2011 Jul-Aug;36(4):390-6.

22. Mortazavi V, Fathi M, Soltani F. Effect of postoperative bleaching on microleakage of etch-and-rinse and self-etch adhesives. Dent Res J (Isfahan). 2011 Winter;8(1):16-21.
23. Nakabayashi N, Kojima K, Masuhara E. The promotion of adhesion by the infiltration of monomers into tooth substrates. J Biomed Mater Res. 1982 May;16(3):265-73.

24. Potocnik I, Kosec L, Gaspersic D. Effect of $10 \%$ carbamide peroxide bleaching gel on enamel microhardness, microstructure, and mineral content. J Endod. 2000 Apr;26(4):203-6.

25. Quitero MFZ, Lopes AO, MATOS, AB. Ensaio de microinfiltração. Rev Odontol UNICID São Paulo. 2012 maioago.;24(2):123-33.

26. Shimaoka AM, Andrade AP, Carvalho RCR. Influência da delimitação de área em estudos laboratoriais para mensuração de resistência adesiva. RPG Rev Pós Grad. 2007 abr.jun.;14(3):249-53.

27. Silveira de Araújo C, Incerti da Silva T, Ogliari FA, Meireles SS, Piva E, Demarco FF. Microleakage of seven adhesive systems in enamel and dentin. J Contemp Dent Pract. 2006 Nov 1;7(5):26-33.

28. Spalding M, Taveira LA, de Assis GF. Scanning electron microscopy study of dental enamel surface exposed to $35 \%$ hydrogen peroxide: alone, with saliva, and with $10 \%$ carbamide peroxide. J Esthet Restor Dent. 2003;15(3):154-64.

29. Tezel H, Ertaş OS, Ozata F, Dalgar H, Korkut ZO. Effect of bleaching agents on calcium loss from the enamel surface. Quintessence Int. 2007 Apr;38(4):339-47.

30. Türkun M, Sevgican F, Pehlivan Y, Aktener BO. Effects of $10 \%$ carbamide peroxide on the enamel surface morphology: a scanning electron microscopy study. J Esthet Restor Dent. 2002;14(4):238-44.

31. Unlü N, Cobankara FK, Altinöz C, Ozer F. Effect of home bleaching agents on the microhardness of human enamel and dentin. J Oral Rehabil. 2004 Jan;31(1):57-61.

32. White DJ, Duschner H, Pioch T. Effect of bleaching treatments on microleakage of Class I restorations. J Clin Dent. 2008;19(1):33-6.

33. Wu J, Donly ZR, Donly KJ, Hackmyer S. Demineralization depth using QLF and a novel image processing software. Int J Dent. 2010;2010:958264.

34. Yamamoto TW, Andrade AP, Shimaoka AM, Carvalho RCR. Efeito de agentes clareadores com diferentes características químicas na microdureza superficial do esmalte dental. RPG. Rev Pós-Grad. 2009 jul.-set.;16(3):127-32.

35. Yazici AR, Keleş A, Tuncer D, Başeren M. Effect of prerestorative home-bleaching on microleakage of self-etch adhesives. J Esthet Restor Dent. 2010 Jun;22(3):186-92. 